\title{
Quality Management System in Fusion Research-Experience From W7-X
}

\author{
Reinhard Vilbrandt, Hans-Stephan Bosch, and Jost-Henrich Feist
}

\begin{abstract}
The execution of quality management (QM) and quality assurance activities in the construction of a fusion device is a very complex task. Nevertheless, $Q M$ is a useful management tool, if we comprehend $\mathrm{QM}$ as the request for continuous reflection on present tasks and on the best way to improve the processes with respect to the scientific and technical aims. Therefore, a quality management system has been introduced for the Wendelstein 7-X construction project. A lot of experience exists in industry but for such one-of-a-kind construction projects of an experimental device, we have to adapt the system to our own requirements which differ and are more complex.
\end{abstract}

Index Terms-Project management, quality, system engineering.

\section{INTRODUCTION}

$\mathbf{T}$ HE use of a quality management system (QMS) is very important also in the field of experimental fusion research. This has become increasingly important, as fusion experiments and devices have become larger and much more complex. With the larger number of people involved within a project, a formal QMS is mandatory, which fixes the rules applicable for everybody. This is even more important within worldwide collaborations of fusion groups, when laboratories and industries with different background and experiences contribute to a common hardware.

However, this means a transition in the working habits for fusion physicists and engineers that were used to work in smaller groups. Because fusion often has special needs and requirements, which are not common to industries, the fusion community cannot always rely on the established norms and standards. We have to introduce our own technical rules to make industries aware of these needs.

\section{ShORT History OF THE W7-X QMS}

In the application for the preferential support from EURATOM for the construction of Wendelstein 7-X (W7-X) in 1995, there was the obligation of the Max Planck Institute for Plasma Physics, Greifswald, Germany (IPP) to apply an industrial type management for this project. Part of this approach was to establish a QM department which had, in the beginning in 1997, less than three members. The first version of a quality management handbook (QMH) was released in 1999. It described the fundamental intention to realize the technical quality according to the state of technology and science [1]. The standard DIN EN ISO 9001:1992 with its 20 quality elements, which was effective at that time, was the basis of this first version. This standard focused on product quality first. The technical requirements had been fixed in the W7-X system specification; and it seemed to be not so much effort to fulfill also the appropriate quality by using some basic rules and quality assurance (QA) measures. The technical quality should not be a problem, if all physicists and engineers would work properly and quality conscious.

However, already during the first years of design and placement of the first orders it became clear that the construction of such an experiment is a very complex challenge where a large number of technical and organizational tasks have to be solved simultaneously. For instance, the level of complexity of orders, which had to be put in the industry to achieve the desired quality for a nearly irreparable device was much higher than thought, because there was, for example, no sufficient experience for manufacturing of composite components for superconducting magnet modules. We had to deepen the work in manufacturing details and processes unexpectedly. We could not simply order components with a certain quality, but we had to increase our in-house involvement in many details of the manufacturing processes dramatically. We had to qualify almost all processes alone or in collaboration with industrial partners. Our field of responsibility increased as well as the complexity of tasks. During this starting period of the project, we recognized that it would be necessary to consider all the necessary processes more in context. Quality means not only the technical quality but also the organization of research and development activities and the documentation, the proper handling of changes and deviations, assignment of responsibilities, and so on.

The product-oriented QA system was changed consequentially to a process-oriented QMS. In the first phase, the activities were focused on single processes, such as handling of orders to be placed, including the writing of specifications, the call for tenders, the assessment of offers, and the supervising of the manufacturing and delivery processes in detailed steps. Supported by some templates these processes could be handled much easier and more effective by the responsible officers.

This transition in thinking was also supported by the new revision of the DIN EN ISO 9001:2000, which was also more focused on processes than on simple QA measures. In 2004, the first process-oriented version of the QMH was issued [2]. 
In addition a new documentation system was installed on a centralized basis. Now it was possible to archive all drafts and documents according to the so called KKS (power plant identification system), a system of assigning all components of a whole industrial facility to a numbering scheme, which was originated by German Vereinigung der Grosskesselbesitzer (VGB) [3].

Step by step, more complex process instructions like handling of quality deviations and handling of change requests (CRs) were created. These process instructions describe in detail the approach, the responsibilities, and the further treatment in every case of occurrence until the final control of measures including proper documentation and assessment of consequences. Special care was necessary to fix the interactions between all colleagues responsible for single components or subsystems which can be influenced by deviations or changes potentially as well as the escalation management. Following the organizational structure of the project the final decisions are made by the chief engineer or the project directors.

In 2007, the complete rearrangement to a process-oriented QMS one could be completed.

To regulate all the work and examining steps during the technically demanding assembly, it seemed useful to take over the manufacturing and control sequence plans which are usual in the industry. Because industrial assembly processes mostly are focused on the repetition of processes with continual results the creation of such a plan is more or less a standard routine. The requirements of such documents during the construction of a prototype like a fusion experiment are more complex. Planning and careful documentation of each step to ensure that all steps are carried out as planned and the envisaged quality will be met are comparable with industrial practice. However, the necessary number of input documents to be used for each step (working instructions, drawings, welding and soldering procedure specifications, change notes, technical guidelines, collision reports, test procedures, etc., with their document and right version number) is much larger than in comparable industrial processes, because often very special and new manufacturing methods are used, which are not part of the standardized industry procedures yet. Also the number of persons in charge and the necessity for an as built documentation with all deviations for traceability to find out causes in case of faults during later operation are more demanding than in routine industrial operation.

Therefore, to handle the rather complex execution of the assembly the so called quality assurance and assembly plan (QAAP)-template had been developed for W7-X, which includes both all the documentation and the organization of interaction with the responsible parties for the single component and QA [4].

Of course, during the long-lasting process of constructing an experiment like W7-X, the main focus of activities changed. Although in the beginning the specification and purchase of the big components dominated the work, next the assembly of the central machine was in the main focus for almost 4 years, now currently the large number of peripheral systems and diagnostics are more important. Whenever it was necessary, the QMS was adapted with respect to new conditions and requirements as well as the experience gained before. For example, 3 years ago a risk assessment for later device operation was introduced. In this process, all available sources like change notes, nonconformity reports and nonconformance tags, and minutes of the upper management or the configuration control board (CCB) are assessed to determine additional risks in comparison with the basic specifications. A central change/deviation database in the project $\mathrm{W} 7-\mathrm{X}$ is used, in which all these issues are collected to avoid negative possible cross effects on other components [5]. Meanwhile, the preparation of the commissioning and the later operation of the experiment are the next important steps.

\section{W7-X QUALITY ORGANIZATION}

As already mentioned, the first conception was a rather small quality department with about three members. This turned out to be unrealistic for a project like W7-X. During a reorganization of the project in 2004, the position of the QM and QA was strengthened and the number of staffs was increased.

Meanwhile, three colleagues of the QM department are busy with mainly organizational tasks. Primarily the check of all documents like specifications, work, and test instructions, QAAP are included as well as investigations of planned measures with respect to quality aspects. The retention and steady improvement of the QMS are also included.

The department has altogether nine qualified QA inspectors with certificates in NDT for specialized tasks. They deal with the enormous amount of quality checks at suppliers and during incoming inspections as well as in-house in the own workshops and labs and during the assembly itself. Almost all of the QA inspectors have certificates in special fields like visual test (VT), dye penetration test (PT), ultrasonic test (UT), radiation test (RT), and leak test (LT) as well as high-voltage tests, electrical tests, and so on. The QA lab is equipped with microscopes, a device for preparation of macroexamination specimen, endoscopes and videoscopes, ultrasonic measurement devices, a lot of mechanical and electrical gauges, and so on to provide all the necessary activities.

Additionally a number of internal inspectors have been appointed. These are experienced colleagues from other departments like vacuum group, metrology or welding supervisors. They support the QA activities by taking over QA examinations. The four-eye principle is always used to check the working results to exclude organizational or operational blindness consistently.

\section{STANDARDS AND RULES}

The entire construction of W7-X has to follow the binding regulations of the authorities. The common requirements according to the European Directive on Machinery, Pressure Equipment Directive, Directive on Safety, and so on, and the requirements due to the special rules of the local authorities with respect to the erection and operation of the experimental device must be fulfilled. 
But what happens with all the national and international standards, like ANSI, ASME, DIN, ISO, European standards EN, and so on, and rules like ITER Design Rules [6], [7], UHV Guidelines like [8], various nuclear safety Standards, for example, German KTA Rules [9], and so on. Should or must we follow them? On one hand, they contain the long-term experience of manufacturing and take also into account the progress by continuous releases; on the other hand, they reflect the common experience and requirements of special branches of industry, for example, chemistry or machine building. Indeed, we cannot find any special standards or rules for fusion research experiments with their new complexity of requirements covering almost all industrial branches.

Nevertheless, the existing standards and rules give a helpful basis for our work. We can use tools and codes from industry for calculations and can adapt and extend them to describe and solve our special tasks. We can use the description of material properties as well as the acceptance criteria and testing procedures. With the standards we can participate on the long-lasting experience of the industry, and we can assess whether our design will be an exceptional challenge or not.

But they do not cover all of our needs; neither in the specialized technical area of fusion nor in the ambitions to work according to the highest standards of science and technology.

Often we did not find equivalent standards which describe our requirements and their realization in manufacturing and testing, because they are not common practice in industry. For instance, the serial manufacturing of the superconducting coils was such a new and complex challenge without former experience and standards. We had to describe almost all the material and functional requirements, tolerances, and appropriate tests ourselves and had to carry out some special development and training processes together with manufacturers to bring them up to the necessary level to fulfill the requirements of W7-X.

Thus, we decided to describe specific requirement and rules in technical guidelines which cover more or less single subjects, and this was found to be the standard situation also in other fusion experiments.

So we compiled a general guideline for the use of materials, which summarizes all the technical aspects and the obligations given by the authority. Similarly, there are also guidelines for welding at W7-X, for ultrahigh-vacuum conditions and the appropriate measures in construction, manufacturing and assembly, and so on. The guidelines are according to the existing standards and rules. The exceptions are described as well as the additional requirements. Of course, also the necessary tests or certificates and documentation are included.

This not only gives a documented summary for these items as mandatory supplements in technical specifications for orders, but also supports the engineers and technicians who work in the project on a steady or temporarily basis.

But we had to recognize that even these technical guidelines are not fully applicable, because national standards and rules are inconsistent. So they hamper the smooth purchase processes of components or materials from foreign suppliers sometimes. The evidence is still inconclusive on this issue, but the experience should be similar also in other projects of fusion research. It seems to be time that the fusion community starts to think about their own standards and rules which describe all the necessities found out during the construction and operation of their experiments.

Finally, from the beginning of concrete planning of DEMO, we need such specialized standards and rules which consider requirements of fusion technology. This also concerns the list of security standards for fusion power stations which must be different from those of the present fission nuclear power industry. It is necessary that these standards will become familiar to industrial partners and authorities as soon as possible.

\section{INTERNAL AND EXTERNAL AUDITS}

Important tools for the steady improvement of the QMS are internal audits. At W7-X these take place biennially to check the awareness of the staff and correctness in following the different processes. The content of the audits is prepared individually, related to the special tasks of each department, by the quality department to minimize the effort and increase the efficiency of the audit. Some members of the quality department have received a special training as certified auditors. In addition to this control function, the project uses these audits to discuss the usefulness and proper work of process and work instruction as well as organizational details in division of labor. The aim is to discover enhancement potentials and capabilities of the system and their adaptation.

The minutes of each audit contain the compliance status that was found and is the basis for a corresponding to-do-list for corrective actions. The results of each single audit are discussed with the team leaders and their boss individually. The fulfillment of the agreed actions is controlled on the agreed due date. On the other hand, all suggestions and advices for improvement of processes or working sequences are collected by the quality department and are summarized in a report on all internal audits to the upper management. Also a to-do-list of measures to be realized is set up in agreement with the upper management and is settled in the next period.

In 2009, we invited the Technischer Überwachungsverein Nord (TÜV) Nord Cert, a notified and accredited serviceprovider for comprehensive certification of management systems, to carry out annual external audits in the project. This outside view of the QMS helps to avoid organizational blindness. Despite of significant differences in the project W7-X compared with conventional industrial or serviceoriented enterprises the TÜV has provided useful advices for our paper by evaluating our processes and practical work. We received the corresponding certificate according to the DIN EN ISO 9001:2008 in 2009 for the first time. It supports the cooperation with the QM in the industry because we can now meet each other at the same professional level.

Since 2009, an annual report to the project management on all quality activities has been given by the head of quality department. The report covers beside the assessment of the main elements of the QMS also an assessment of so-called weak factors like responsibilities, problem analysis and countermeasures, knowledge and experience transfer, 
motivation, and satisfaction of employees. The report is the basis for an annual management review of the whole QMS, which results in concrete tasks for improvement of the system regularly.

\section{Project Regulation and Control}

It must be emphasized again that QM not only includes all measures to ensure the technical quality but all processes of the project. The project management is therefore also included as an important task.

Different from the standard industry production with prototypes, small Series, and final series production, the construction of a unique fusion experimental device like W7-X implies some specifications. On one hand, there is a tremendous complexity; on the other hand, there is no continuous development but an order of magnitude jump in complexity carried out by people with no or little experience. Remember the last construction of a fusion device in IPP has been three decades ago. Nevertheless, the project has planned steps and aims at a technical realization according to the specification within a limited budget and given due dates. What one can take from industry is the structuring of the project and the well-planned approach with respect of working packages and steps and resources.

A very effective system has been introduced within the W7-X project for the integrated budget and schedule planning and controlling of each subproject (integrated planning tool). Only this extended planning and controlling of work packages on basis of detailed and linked work breakdown structures of all departments provides the chance to synchronize all the activities and due dates.

In general, a clear structuring of the project in subprojects with a clear assignment of responsibilities is the basis for good project management.

For risk-prone processes contingencies were implemented from the beginning, more than usually accepted in industry to provide corresponding planning reliability. These are both temporal buffers and financial management, but in some cases also a plan B is set up for the case that an intended solution proves to be unrealizable.

Supported by a continuous reporting and decision process on a weekly basis, it allows the project to react early and efficiently to deviations and turbulences.

To measure the long-lasting performance in consumption of finances and time against construction progress also tools like earned value management are used [6].

\section{Assessment of the System Performance}

The measurement of the system performance related to technical factors or quality deviations is rather different from the situation in industry. Because most of development and test processes as well as assembly steps are really unique, the assessment cannot use statistical methods. Despite of all carefulness during the design and manufacturing, modifications and quality deviations are unavoidable. In all these processes, potential risks have to be identified and assessed in detail, because often it is not possible to repeat the processes
TABLE I

QA EFFORT UP TO NOW (ONLY IN House)

\begin{tabular}{|l|r|}
\hline \multicolumn{2}{|c|}{ QA effort up to now (only in house) } \\
\hline total no. of tests (estimated) & 164,000 \\
separate test protocols & 26,300 \\
(other confirmed in QAAP) & 112,000 \\
visual tests (incl. videoscopy) & 3,800 \\
(fluorescent) dye penetrant tests & 3,400 \\
X-ray tests & 14,400 \\
leak tests & 11,000 \\
ultrasonic tests for fasteners & 400 \\
ultrasonic tests for weld seams & 15,000 \\
electrical tests & 2,200 \\
microscopy (macro-examination specimen) & 1,800 \\
incoming tests of material & 3,850 \\
\hline no. of nonconformity tags & 1,900 \\
no. of non conformity reports & 1,050 \\
no. of change notes &
\end{tabular}

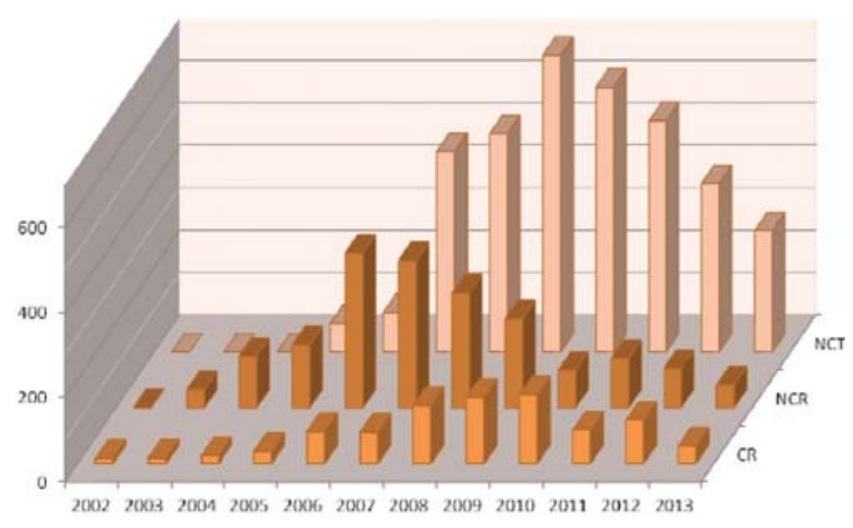

Fig. 1. Number of NCTs, NCRs, and CRs per annum.

or sometime it is not possible to improve it. Therefore, all changes to technical specifications and all quality deviations during the manufacturing or the assembly process are subject to a formal assessment process evaluating possible effects on W7-X. The assessment regularly includes the influence on the scientific goals, the properties of the component itself as well as on the schedule, costs, and quality aspects before release. In this process, sometimes also certain risks must be taken for effort reasons. But, it is always a controlled process and the final decision is always made by the upper management. The number of changes which was not expected at the beginning of the project shows the necessity of a well organized and operating system.

Some facts in Table I illustrate the huge amount performed until now only in the assembly of W7-X.

Figs. 1 and 2 show the number of CRs, nonconformity reports (NCRs), and nonconformity tags (NCTs) and the 


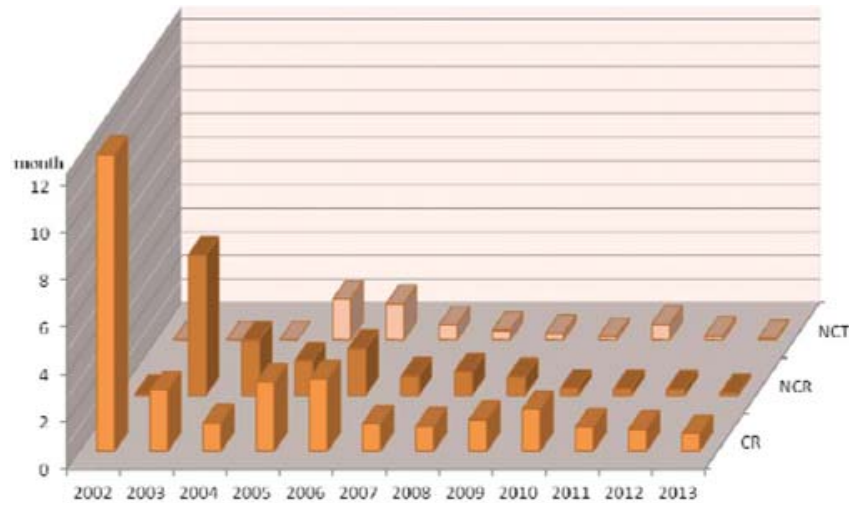

Fig. 2. Mean time until approval of the measures.

duration between occurrence and the approval of change notes or measures for the elimination of quality deviations, which could be shortened fundamentally by acting along the accepted process instructions.

\section{PREPARATION OF COMMISSIONING AND OPERATION}

The commissioning of W7-X will start in spring 2014. This sequence must be planned now, using the experience of the past as much as possible [11], [12]. The starting point was a workshop with participants from other fusion experiments or large accelerators from the international community in January 2013. They reported about the practice of the commissioning, their organizational structures and special methods. In principle, the project will use again the already familiar procedures like process- and work-instructions and handling of quality deviations and changes. Some working templates like Commissioning Assurance Template (CAT) will be used, which is comparable with the well-known QAAP in the assembly phase but with special adjusted issue and form, and special test procedures will complete the set of documents. As commissioning will be the basis of later operation, special emphasis must be put on proper documentation and transfer of experience. Of course, for all local or integrated commissioning of plant components or whole systems, we also need a detailed time and resources planning like a WBS to manage the increasing complexity. It must contain the internal and external preconditions from other components in detail as well as the preparation of the necessary documents on time. Furthermore all planned inspections before commissioning, the main steps of commissioning, and the documentation have to be planned accurately for sufficient interaction of all participants in these processes. This plan must be carefully checked by the involved persons in charge and must be released by the commissioning board. The separate CAT for each commissioning must contain the single steps and tests with their necessary input documents like working and test instructions, detailed adjustments, and operation points as well as the responsible persons who have to interact and the output documentation of each step.

\section{CONCLUSION}

The complex performance of all QM and QA activities in the project $\mathrm{W} 7-\mathrm{X}$ is not an easy but a very complex task.
If we understand QM as a request for a continuous reflection on present tasks and their realization and on the best way to improve and reorganize the processes with respect to the prospective scientific and technical aims, it is and will be a useful management tool. We can learn a lot from industry, but we have to adapt the industrial QM system and all concrete measures to our own requirements which sometime differ and are even more complex.

\section{ACKNOWLEDGMENT}

All the aforementioned works as were done in close cooperation with the departments of W7-X, the assembly staff, project control, and the QM-department. The good collaboration between these various partners, sometimes under strong time pressure, is gratefully acknowledged.

\section{REFERENCES}

[1] J.-H. Feist et al., "Quality management for WENDELSTEIN 7-X," Fusion Eng. Design, vols. 58-59, pp. 809-813, Nov. 2001.

[2] J.-H. Feist et al., "Quality management for WENDELSTEIN 7-XLessons learned," Fusion Eng. Design, vol. 82, nos. 15-24, pp. 2838-2843, Oct. 2007.

[3] KKS Power Plant Identification System, 7th ed. Essen, Germany: VGB PowerTech e.V., 2010.

[4] R. Vilbrandt and W7-X Team, "Quality assurance during assembly of Wendelstein 7-X," Fusion Eng. Design, vol. 86, nos. 6-8, pp. 655-658, Oct. 2011.

[5] R. Vilbrandt, H.-S. Bosch, and P. van Eeten, "Risk management as an executive task in the construction of Wendelstein 7-X," Fusion Eng. Design, vol. 88, nos. 9-10, pp. 2151-2154, 2013.

[6] Structural Design Criteria for ITER, General Section (SDC-G), document ITER G 74 MA 6 01-05-28 W0.4, 2001.

[7] Structural Design Criteria for ITER In-Vessel Components (SDC-IC), document ITER G 74 MA 8 R0.1, 2001.

[8] UHV Guidelines for X-Ray Beam Transport Systems, document XFEL.EU TN-2011-001-03, Jun. 2013.

[9] KTA Program of Standards, The Nuclear Safety Standards Commission, Salzgitter, Germany, 2001.

[10] A. Lorenz, H.-S. Bosch, and K. Kuttler, "Implementation of earned value management tools in the wendelstein 7-X project," in Proc. IEEE/NPSS 24th Symp. Fusion Eng. (SOFE), Jun. 2011, pp. 1-4.

[11] T. Klinger et al., "Towards assembly completion and preparation of experimental campaigns of Wendelstein $7-\mathrm{X}$ in the perspective of a path to a stellarator fusion power plant," Fusion Eng. Design, vol. 88, nos. 6-8, pp. 461-465, 2013.

[12] H.-S. Bosch, R. Brakel, M. Gasparotto, D. A. Hartmann, R. Herrmann, and D. Naujoks, "Preparation of the Wendelstein 7-X commissioning," in Proc. 25th Symp. Fusion Eng. (SOFE), Jun. 2013, pp. 1-4.

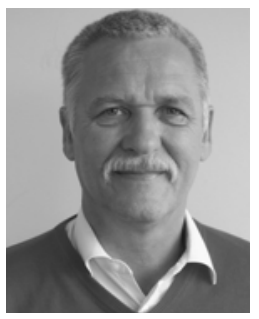

Reinhard Vilbrandt received the Dipl.-Ing. degree in technical cybernetics and automation engineering from the University of Rostock, Rostock, Germany, in 1982, and the Dr.Ing. degree in computer science from the University of Seafaring, Rostock, in 1986.

He was a Scientist of Apparatus Engineering and Research Technologies with the German Academy of Sciences, Berlin, Germany, where he was also the Head of Research and Development in the power plant industry. He is currently the Head of the Quality Management of the Wendelstein 7-X Project with the Max Planck Institute for Plasma Physics, Greifswald, Germany. 


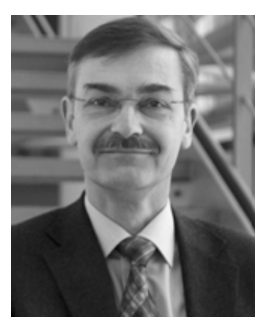

Hans-Stephan Bosch received the Dipl.-Phys. degree from Ludwigs-Maximilians University, Munich, Germany, in 1983, the Dr.rer.nat. degree from the Technical University of Munich, Munich, in 1986, and the Habilitation degree from the Humboldt University of Berlin, Berlin, Germany, in 2000.

He has been a Lecturer with the University of Greifswald, Greifswald, Germany, since 2008. He is currently the Associate Director of the Wendelstein 7-X Project and the Division Head of W7-X Operations with the Max Planck Institute for Plasma Physics, Greifswald. His current research interests include plasma edge and divertor physics.

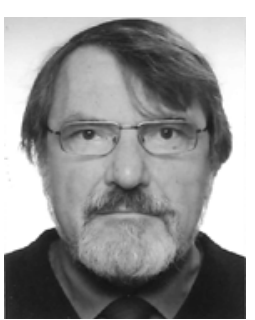

Jost-Henrich Feist received the Dipl.-Phys. and Dr.rer.nat. degrees from the University of ErlangenNuremberg, Erlangen, Germany, in 1972 and 1975, respectively

He joined the Max Planck Institute for Plasma Physics, Garching, Germany, in 1976, and was the Head of the Quality Management of the Wendelstein 7-X Project with the Max Planck Institute for Plasma Physics, Greifswald, Germany, from 1997 to 2010. 